\title{
Characterization of Aircraft Structural Damage using Guided Wave Based Finite Element Analysis for in-Flight Structural Health Management
}

\author{
Banavara R. Seshadri ${ }^{1}$ \\ National Institute of Aerospace, Hampton, VA 23666, U.S.A \\ Thiagarajan Krishnamurthy ${ }^{2}$ \\ NASA Langley Research Center, Hampton, VA 23681, U.S.A \\ and \\ Richard W. Ross ${ }^{3}$ \\ NASA Langley Research Center, Hampton, VA 23681, U.S.A
}

\begin{abstract}
The development of multidisciplinary Integrated Vehicle Health Management (IVHM) tools will enable accurate detection, diagnosis and prognosis of damage under normal and adverse conditions during flight. The adverse conditions include loss of control caused by environmental factors, actuator and sensor faults or failures, and structural damage conditions. A major concern is the growth of undetected damage/cracks due to fatigue and low velocity foreign object impact that can reach a critical size during flight, resulting in loss of control of the aircraft. To avoid unstable catastrophic propagation of damage during a flight, load levels must be maintained that are below the load-carrying capacity for damaged aircraft structures. Hence, a capability is needed for accurate real-time predictions of safe load carrying capacity for aircraft structures with complex damage configurations. In the present work, a procedure is developed that uses guided wave responses to interrogate damage. As the guided wave interacts with damage, the signal attenuates in some directions and reflects in others. This results in a difference in signal magnitude as well as phase shifts between signal responses for damaged and undamaged structures. Accurate estimation of damage size and location is made by evaluating the cumulative signal responses at various pre-selected sensor locations using a genetic algorithm (GA) based optimization procedure. The damage size and location is obtained by minimizing the difference between the reference responses and the responses obtained by wave propagation finite element analysis of different representative cracks, geometries and sizes.
\end{abstract}

\section{Introduction}

The development of validated multidisciplinary Structural Health Management (SHM) system tools, technologies, and techniques to enable detection, diagnosis and prognosis in the presence of adverse conditions during flight will provide effective solutions to deal with safety related challenges facing next generation aircraft. The adverse conditions include loss of control caused by environmental factors, actuator and sensor faults or failures, and damage conditions. A major concern in these structures is the growth of undetected damage (cracks) due to fatigue and low velocity foreign object impacts that can reach a critical size during flight, resulting in loss of control of the aircraft. Hence, development of efficient methodologies to determine the location, and severity of damage in critical structural components is highly important in developing efficient structural health management systems. Approaches for determination of damage size and location in structures can be characterized as either global approaches or local approaches. In the global approach, changes in vibration properties caused by damage are used to detect size and location [1-2]. The presence of damage is indicated by

\footnotetext{
1 Senior Research Scientist

2 Aerospace Engineer, Durability, Damage Tolerance \& Reliability Branch, Fellow, AIAA

3 Aerospace Engineer, Research Directorate
} 
the changes in the frequency response functions (FRF) of a structure. However, when defects are small compared to the dimensions of a structure, it is not easy to distinguish the differences in the vibration response. Sometimes the differences may be below the noise level. Even though techniques have been developed to amplify the differences and eliminate noise, effectiveness of using the vibration approach in detecting small defects in complex structures remains uncertain at present. In the local approach, the use of non-destructive evaluation (NDE) methods for the detection or identification of cracks at an early stage, especially incipient buried microcracks, is very important for ensuring structural safety and integrity of aircraft, nuclear power plants, chemical plants and refineries [3]. Dye penetrant inspection is a traditional low-cost NDE tool that has been widely applied for detecting cracks in components used in many fields, but the technique requires direct access to the component and is unable to detect cracks hidden or buried below surfaces. Another popular and powerful NDE tool, the conventional ultrasonic testing method [4-5], has been extensively used to detect and measure the volume of defects such as buried cracks, corrosion, or voids. The changes in the characteristics of ultrasonic waves propagating across damage are measured and analyzed to detect and characterize damage [6-7]. However, conventional ultrasonic inspection methods using longitudinal or shear waves are time-consuming and inefficient when dealing with large-scale structures because inspection is usually performed in a point-by-point manner. Even with the continuous advancement in global and local approaches, there are large uncertainties associated with the determination of damage size and the location. The uncertainties come from both the method employed and the instrumentation, which results in low confidence in estimating the damage size and location. Hence, there is a need to increase the level of confidence in detecting damage size and location using advanced technologies. In the past, the authors used a combination of strains estimated at a few select sensor locations to simulate strains that would be measured by an on-board Integrated Vehicle Health Management (IVHM) system with either a finite element method or extended finite element method (XFEM) analysis technique to characterize the damage size and location [8-9]. It is necessary that both loading and boundary conditions are known to apply the methods in references 8 and 9. When dealing with real structures, it is not always possible to know the applied boundary and loading conditions. One way to increase confidence in determining damage size and location is to use a guided (Lamb) wave technique. Unlike other approaches, the guided wave approach is useful for detecting and characterizing hidden defects since the wave length is smaller than the defects [10] and can propagate over a large distance in plates and shells [11] making it possible to inspect large areas quickly and efficiently. Active Structural Health Management (SHM) systems using interrogative Lamb waves can cover large areas from one single location. Such systems can be cost-effective and efficient [11]. Another advantage is that Lamb waves provide through-the-thickness interrogation which allows detection of internal defects in thin materials [12]. In a thin plate, the boundaries of the structure interact with waves, causing successive reflections, refractions and mode conversions that create Lamb waves via a complex mixture of constructive and destructive interference. They have been widely used in structural inspection and crack detection of thin structures. Many studies have been conducted on the interaction of Lamb waves with cracks [13-16]. Recently, an experimental investigation into the use of nonlinear Lamb waves for evaluating fatigue micro-cracks was carried out [17]. Soshu and Toshihiko [18] used nonlinear longitudinal waves to detect a closed crack. Shen and Giurgiutiu [19-20] adopted FEM to simulate the interaction between nonlinear Lamb waves and a surface crack in a plate. Wan et al. [21] used finite element analysis to simulate the interaction between nonlinear Lamb waves and a buried micro-crack perpendicular to the upper and lower surfaces of a thin metallic plate. A pitch and catch approach was applied in the FEM model, using two symmetric piezoelectric ceramic transducer wafers as transmitters to generate a single lowest order symmetric mode signal, $\mathrm{S}_{0}$, and a single Piezoceramic Lead Zirconate Titanate (PZT) wafer as the receiver. The simulation results show that the nonlinear Lamb wave technique is indeed capable of detecting a micro-crack in a thin plate. This allows us to propose a baseline-free indicator to identify and detect a micro-crack in a thin plate.

In the past decade, there has been increasing interest in the study of sensors that employ ultrasonic Lamb waves. Lamb waves can be excited and detected by a variety of methods. The wedge method is probably the most well-known, in which a prism or wedge is employed to convert bulk waves into Lamb waves. Other transducer techniques have been reported in the literature, including optical methods, electromagnetic acoustic transducers (EMATs), capacitance transducers, and air-coupled transducers. Recent work using lasers for both generation and detection is potentially very attractive. This laser ultrasonic technique has the advantage of being non-contact and scanned easily and rapidly over the surface of an object, though the required equipment is bulky and expensive and has mainly been used in the laboratory rather than in industrial testing applications. In the majority of standard ultrasonic testing, piezoelectric transducers remain the most utilized for guided wave excitations, since the manufacture and use of these transducers are well understood. Piezoelectric transducers can overcome the above disadvantages of the conventional transducers, and additionally, they are relatively cheap and light weight [22]. 
Piezoelectric transducers are used for both actuating and sensing Lamb waves. Thin disks made from ceramic material PZT are used as the piezoelectric transducers. When transducers are attached to a test specimen for SHM, a transducer that sends out a wave is commonly referred to as an actuator, while a transducer that receives a wave is referred to as a sensor. Piezoelectric transducers are polarized such that a voltage differential applied across the thickness of the disk causes the radius of the disk to contract or expand. The radial displacement creates an elastic wave within the host material that propagates through a medium and eventually strains a sensor. A gain property relates strain to voltage, thus the resulting compression and expansion of the sensing transducer generates voltage differentials which are recorded as voltage amplitudes over time [4]. Recent developments in piezoelectric transducer wafer technology offer the ability to obtain densely distributed sensor responses with little weight addition to a structure [4]. The sensor response at various locations from piezoelectric transducer wafer technology can be used to characterize damage size and location for Structural Health Management (SHM). The current research uses the sensor response at selected points in the structure along with wave propagation response of damaged structure to characterize the damage location and size.

In this paper, a damage characterization method using a wave propagation technique based on finite element analysis for SHM is proposed. A conceptual illustration of SHM similar to the central nervous system is shown in Figure 1. By measuring response at certain specific locations in the central nervous system, diagnostics for any ailment or disorder is accomplished. Likewise, by measuring or estimating structural response at selective locations, damage diagnostics throughout the structure is established. First, a brief introduction to guided wave propagation analysis is discussed. Then, an optimization procedure along with wave propagation-based FE analysis is presented to estimate the damage size and location. Next, several numerical examples are illustrated to demonstrate the effectiveness of the method.

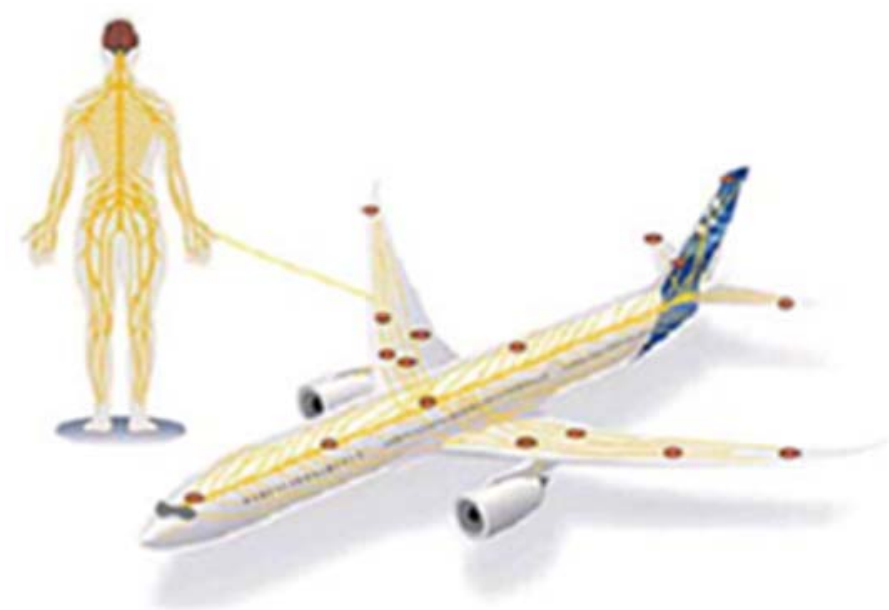

Figure 1. A Conceptual illustration comparing the human central nervous system and an aircraft SHM system.

\section{Guided Wave Propagation Analysis}

This section briefly introduces the basic finite element model development and criterion that are essential for successful guided wave propagation analysis. To obtain adequate accuracy and high efficiency, a meshing strategy with varying mesh density was adopted. In general, a denser mesh will give a more accurate result, but will also cost more in terms of calculation time and computer resources.

\section{A. Finite Element Model}

Three-dimensional finite element models were developed and dynamic simulations were performed using Abaqus $^{\circledR} /$ Explicit [23] software. In this work, specimens analyzed are made of 2024-T3 aluminum and the material 
properties used are shown in Table 1 . The dimensions of the plate analyzed are shown in Table 2. A Hanning windowed tone-burst consisting of five and a half cycles at a frequency of $50 \mathrm{kHz}$ was used as the excitation signal. The temporal waveform is plotted in Figure 2 and adopted the maximum element size and time step to ensure accuracy from the reference [24-25]:

Table 1: The material properties of the plate

\begin{tabular}{|c|c|c|c|}
\hline Density, $\rho$ & Elastic Modulus, E & Shear Modulus, $\mathrm{G}$ & Poisson's Ratio, $v$ \\
\hline $2.768 \mathrm{~g} / \mathrm{cm}^{3}$ & $69 \mathrm{GPa}$ & $28 \mathrm{GPa}$ & 0.33 \\
\hline
\end{tabular}

Table 2: Dimension of the aluminum plate

\begin{tabular}{|c|c|c|}
\hline Thickness, $\mathrm{d}$ & Width, $\mathrm{w}$ & Length, $\mathrm{h}$ \\
\hline $1.0 \mathrm{~mm}$ & $150.0 \mathrm{~mm}$ & $300.0 \mathrm{~mm}$ \\
\hline
\end{tabular}

The five and a half cycle Hanning-window excitation signal is used in this study because it has the following advantages. First, it reduces the energy at frequencies other than the excitation or 'center' frequency. Second, since the signal is based on a limited cycle sinusoidal tone burst, the undesired reflections between packets are reduced. Figure 2 illustrates 5 1⁄2 cycle Hanning-window excitation signals at $50 \mathrm{kHz}$.

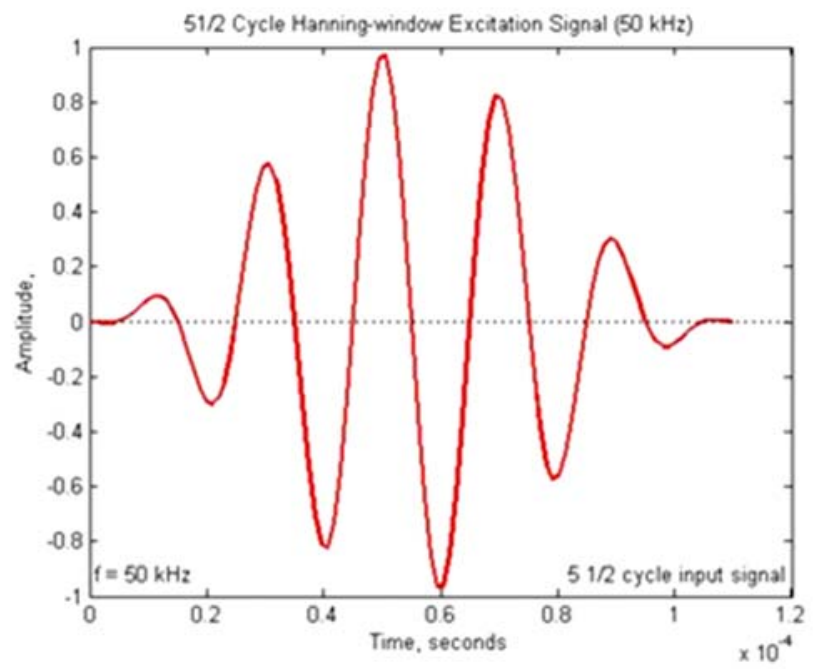

Figure 2. 51/2 cycle Hanning-window excitation signal at $50 \mathrm{kHz}$.

\section{B. Determining Element Edge Length $\left(L_{e}\right)$ of FE Models}

The lowest phase velocity (i.e., transverse wave speed), $\mathrm{C}_{\mathrm{T}}$, and hence the shortest wavelength sets the maximum permissible grid spacing that must be chosen so that spatial aliasing due to the finite element discretization does not occur. The size of the elements is chosen in a manner so that the propagating waves are spatially resolved [24-25]. To do this, more than 10 nodes per wavelength, $\left(\lambda^{1}\right)$ is adequate. To decide on a proper element size, the following procedure is incorporated. First, $C_{T}$ is calculated using equation (1) where $g$ is gravitational acceleration.

$$
c_{T}=\sqrt{\frac{G g}{\rho}}
$$


Simulations have been performed up to frequencies of $300 \mathrm{kHz}$. This maximum frequency, $\left(f_{\max }\right)$ is from the restriction of the frequency-thickness product of the specimen [24-25]. Using $f_{\max }$ of $300 \mathrm{kHz}$ and $\mathrm{C}_{\mathrm{T}}$, the minimum wavelength $\left(\lambda^{1}{ }_{\min }\right)$ is calculated [24-25]:

$$
\lambda_{\min }^{1}=\frac{C_{T}}{f_{\max }}
$$

From the above equation, the typical edge length, $L_{e}$ can be estimated [24-25];

$$
L_{e}<\frac{\lambda_{\min }^{1}}{20}
$$

This relation ensures that there are at least 20 elements across the smallest wavelength of interest. Rounding down the value of $\mathrm{L}_{\mathrm{e}}$ to a value of $3 \mathrm{~mm}$ ensures that an integer number of elements can be placed along the length and width of the plate in the finite element model. An edge length of $3 \mathrm{~mm}$ is used in the mesh module in ABAQUS/CAE [23]

\section{Calculating Time Step $(\Delta t)$}

The use of an adequate integration time step $(\Delta t)$ is very important for the accuracy of the solution. In general, the accuracy of the model can be increased with increasingly smaller integration time steps. With time steps that are too long, the high frequency components are not accurately resolved. On the other hand, time steps that are too small can waste calculation time. Therefore, a compromise must be found. For this study, 20 points per cycle for the highest frequency has been estimated to be a reasonable compromise. This gives accurate solutions in an efficient manner [24-25]. To calculate the time step $(\Delta t)$, first the Lame's constants $(\mu, \lambda)$ need to be calculated from equation (4) and (5), respectively,

$$
\begin{aligned}
& \mu=\frac{E}{2(1+v)}=G \\
& \lambda=\frac{E \gamma}{(1+v)(1-2 v)}
\end{aligned}
$$

Using the result of Lame's constants $(\mu, \lambda)$ above, the longitudinal wave speed $\left(C_{L}\right)$ can be calculated from equation (6),

$$
C_{L}=\sqrt{\frac{(\lambda+2 \mu) g}{\rho}}
$$

The time step $(\Delta t)$ has two criteria from equation (7) and (8). Equation (7) is the CFL condition (named for Courant, Friedrichs and Lewy). This condition prevents a longitudinal wave from traveling completely through an element during a single time step. Equation (8) is the criteria given by Moser [25]. Using the minimum element edge length $\left(\mathrm{L}_{\mathrm{e}}\right)$ and a maximum frequency $\left(f_{\max }\right)$ of $300 \mathrm{kHz}$, both of the criteria can be calculated [24-25];

$$
\begin{gathered}
\Delta t<\frac{L_{e}}{C_{L}} \\
\Delta t_{\max }=\frac{1}{20 f_{\max }}
\end{gathered}
$$


Equation (8) ensures that there are at least 20 time steps during the cycle of a wave at the highest frequency. A $5.0 \mathrm{~ns}$ time step $\left(\Delta \mathrm{t}_{\max }=5 \mathrm{~ns}\right)$ meets these criterion and the value is used in the step module in ABAQUS CAE [23].

\section{Objective Function and Optimization Procedure}

\section{A. Objective Function}

For the present study, the damage is assumed to be in the form of a crack in a plate with width and height as shown in Figure 3. The damage is characterized by three independent variables as shown in the figure. The first two variables are the location of the center of the damage, $X_{r}$ and $Y_{r}$, and the third variable is the damage size $2 a_{r}$. The three independent variables define the damage configuration in this paper.

Consider an arbitrary reference damage in a plate as shown in Figure 4. It is assumed that the response from pre-selected sensor locations is known for this reference damage configuration and is called the reference solution in this paper. The sensor and actuator locations are shown schematically in Figure 4. The actuator is located at a fixed distance, $A_{L}$, from the bottom of the plate. Sensors are positioned at a fixed distance away from the edges of the plate. In the optimization procedure, the damage location, $X_{r}$ and $Y_{r}$ and damage size, $2 a_{r}$ are treated as

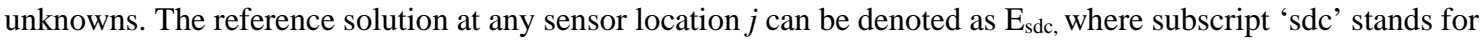
signal difference coefficient. The process of estimating the reference damage configuration begins by creating a finite element model with an initial assumed damage configuration with size $2 a_{i}$ and location, $\left(X_{i}, Y_{i}\right.$, $)$ as shown in Figure 5. The finite element mesh is not shown in Figure 5 for clarity.

An optimization procedure must then be used to iterate the damage configuration to minimize the objective function. A wave propagation analysis is performed at each iteration, and the responses at the sensor locations are computed. Let the response at the $j^{\text {th }}$ sensor location from the wave propagation analysis at the $i^{\text {th }}$ iteration be denoted as, $\mathrm{S}_{\mathrm{sdc}}$. If the damage configuration for the current iteration matches the reference damage configuration, the response at all sensor locations will be identical. If the reference response is not the same as the wave propagation response for the current damage configuration at all the sensor locations, another finite element model is built with a new assumed arbitrary damage configuration. In order to automate and reduce the number of iterations needed to match the response from the reference solution and the arbitrary configuration, an optimization procedure is utilized:

1. Start the optimization procedure with an arbitrary damage location and size. Construct a finite element model for the current assumed damage configuration.

2. Perform a wave propagation analysis for the current damage configuration and compute response at all the sensor locations, $N$.

3. Find the sum of the root square error between the reference response, $\mathrm{E}_{\mathrm{sdc}}$ and the computed response, $\mathrm{S}_{\text {sdc }}$ over all the sensor locations from $\mathrm{j}=1$ to $N$ and integrated over time period, $\mathrm{t}$ as,

$$
\phi=\int_{0}^{t}\left[\sum_{j=1}^{N}\left(E_{s d c}-S_{s d c}\right)^{2}\right]^{1 / 2} d t
$$

where $\phi$ in equation (9) is generally referred as an objective or error function in the optimization procedure.

4. When the estimated value for the objective function in Equation (9) is less than a pre-selected tolerance (ideally it should be exactly zero), convergence is achieved and the iteration is stopped. Then, the current damage configuration is the estimated damage configuration.

5. If convergence is not achieved, the optimization algorithm will estimate a new damage configuration and steps 2-4 are repeated until convergence is achieved. 
In the analysis, the actuator is simulated by using the Hanning excitation signal shown in Figure 2 as a prescribed displacement boundary condition. The displacements obtained at the sensor locations are treated as displacements measured by an on-board IVHM system. This iterative process can be automated to reduce the number of iterations needed to match the response from the reference solution and the arbitrary configuration using an optimization procedure.

The estimation of the damage configuration using the optimization procedure is demonstrated through numerical examples in subsequent sections. The following numerical examples are presented:

a) A damage configuration in which $X_{r}$ and $Y_{r}$, are unknown.

b) A damage configuration in which $X_{r}$ and $Y_{r}$, and 2a are unknown.

The accuracy and reliability of estimating the damage configuration are largely dependent on the number of sensor locations at which responses are available for the structure. In general, the larger the number of sensors, the greater the accuracy in detecting the damage configuration.

\section{Numerical Examples}

The developed capability is demonstrated on damage fully contained within a two-dimensional plate. The material properties and plate geometry are shown in Tables 1 and 2, respectively. It is necessary for each iteration in the optimization procedure that a new finite element model with the current damage configuration be created.

\section{A Genetic Algorithm (GA) Based Optimization Study:}

To minimize the objective function defined in Equation (10), an optimization procedure must be used. In this section, the capability of the GA based technique is investigated to minimize the objective function, i.e. to estimate the damage configuration. The constraint based GA routine available in MATLAB is used to minimize the objective function in this study [26].

The genetic algorithm (GA) implemented in Matlab [26], was investigated since it is a method for solving highly nonlinear objective functions and is globally convergent, as opposed to the gradient-based technique, which converges to a local minima. Unlike the gradient-based technique, which generates a single trial point at each iteration, the GA generates a population of points at each iteration. The GA then selects those trial points from the population, i.e. most closely matches the target solution, and generates a new population of points based on the fittest members.

Some potential downsides of the GA are:

- $\quad$ Since the trial points in the first iteration are randomly generated, there is no guarantee that the optimization will produce equivalent converged solutions upon repeated trials;

- $\quad$ there are several input options that must be provided and those that provide the fastest, most consistent convergence must be determined by trial-and-error and;

- $\quad$ more function calls are required (in this case, wave propagation analyses).

It was found that the following GA-input options provided consistent convergence behavior for the problem under investigation:

- $\quad$ Population size: $20-60$

- Creation function: Feasible population

- Selection: Stochastic uniform

- Mutation: Adaptive feasible

- Crossover: Scattered 
This population size was large enough to provide sufficient variation diversity, while minimizing the number of function calls required. By using the 'Feasible population' Creation function and 'Adaptive feasible' Mutation, the trial points were guaranteed to stay contained within the upper and lower bounds on the damage configuration. This was important because damage configurations that lie outside the domain would potentially create damage that was not fully contained within the plate, which were considered invalid possibilities in this study. Tournament Selection chooses parents to the next generation by randomly sampling the current population and keeping only the fittest. One crucial behavior that these inputs provide is the ability to maintain diversity in the population after several iterations.

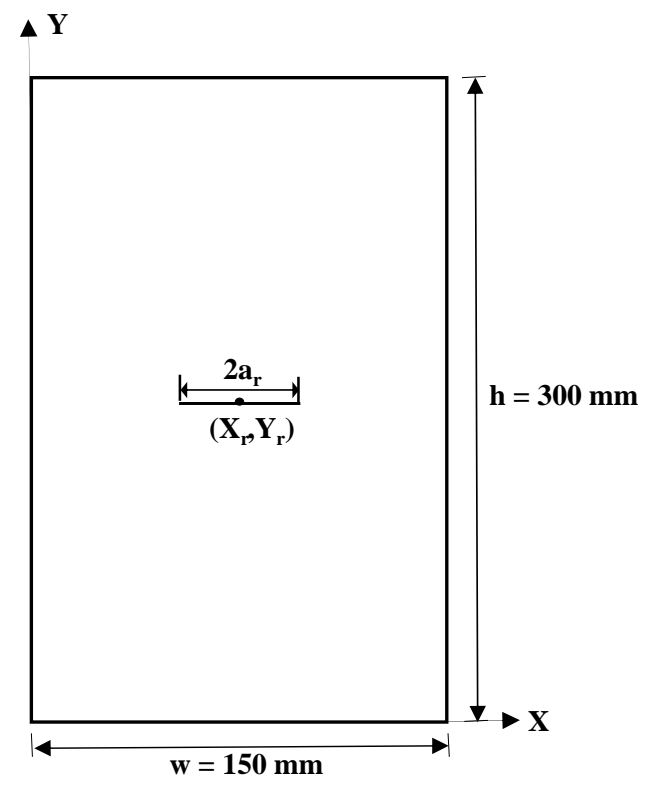

Figure 3. Schematic of geometry analyzed and three variables used to define the damage configuration.

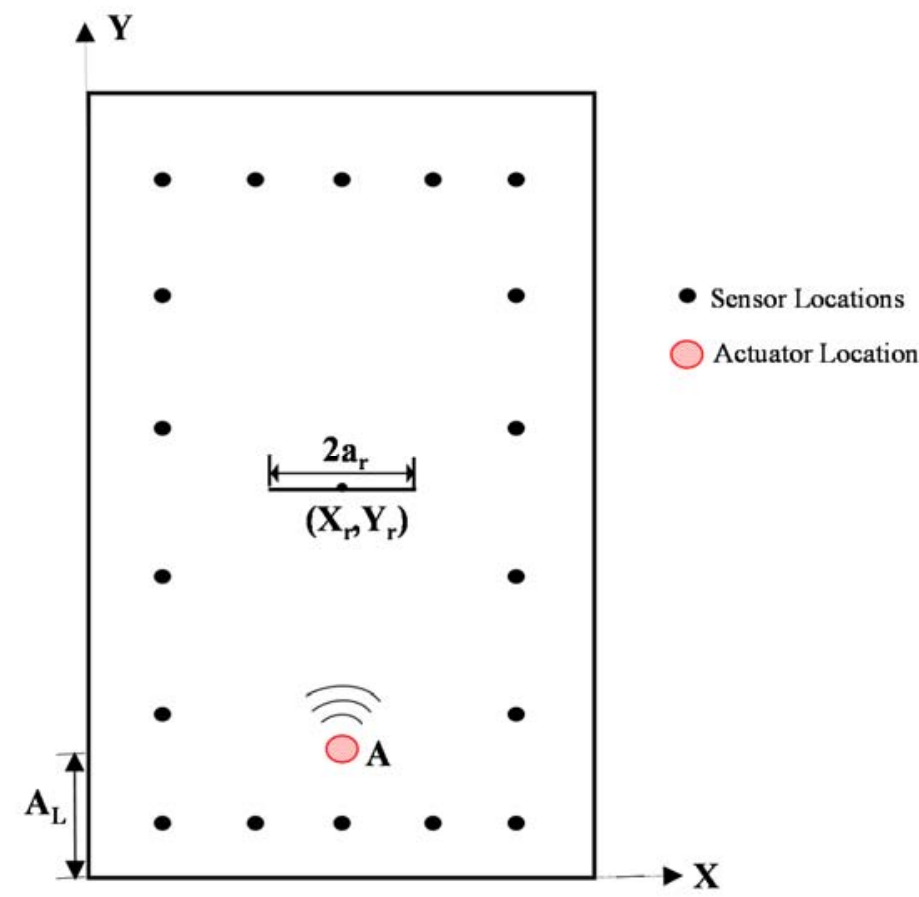

Figure 4. Schematic of damage configuration and sensor and actuator locations.

8

American Institute of Aeronautics and Astronautics 


\section{B. Reference Solution Generation:}

The responses at the sensor locations are the only input to the optimization procedure discussed in the previous section. For this paper, response data from pre-selected sensor locations are generated by performing a wave propagation analysis of the reference damage configuration. The responses at the sensor locations are interpolated from the nodal displacement in the wave propagation analysis. The response data obtained for the reference damage configuration are used as the reference solution in the optimization procedure.

Three reference damage configurations were considered for the numerical examples presented in this paper and are shown in Figure 6. Case I is a center damage configuration, while Case II is an upper right damage configuration. Case III is a bottom left damage configuration.

For Case I (center damage configuration), the reference location is $X_{r}=75 \mathrm{~mm}, Y_{r}=150 \mathrm{~mm}$, and the damage size is $2 \mathrm{a}_{\mathrm{r}}=25 \mathrm{~mm}$.

For Case II (upper right damage configuration), the reference location is $\mathrm{X}_{\mathrm{r}}=100 \mathrm{~mm}, \mathrm{Y}_{\mathrm{r}}=225 \mathrm{~mm}$, and the damage size is $2 \mathrm{a}_{\mathrm{r}}=25 \mathrm{~mm}$.

For Case III (bottom left damage configuration), the reference location is $X_{r}=50 \mathrm{~mm}, Y_{r}=75 \mathrm{~mm}$, and the damage size is $2 \mathrm{a}_{\mathrm{r}}=25 \mathrm{~mm}$.

\section{Damage Location as the Unknown Variable}

Next, the optimization procedure is demonstrated in the example with two unknown variables. The damage location, $X_{r}$ and $Y_{r}$, were treated as the two unknowns in the problem. For this example, the reference Case I, the selected damage configuration is shown in Figure 6. The processes of invoking MATLAB ${ }^{\circledR}$ GA optimization routine, generation of finite element model using ABAQUS CAE, wave propagation analysis using ABAQUS EXPLICIT, processing of the results file for sensor response data using ABAQUS VIEWER, and comparison of sensor response data with the reference solution in the evaluation of the objective function is all automated and carried out using PYTHON scripting language [27].

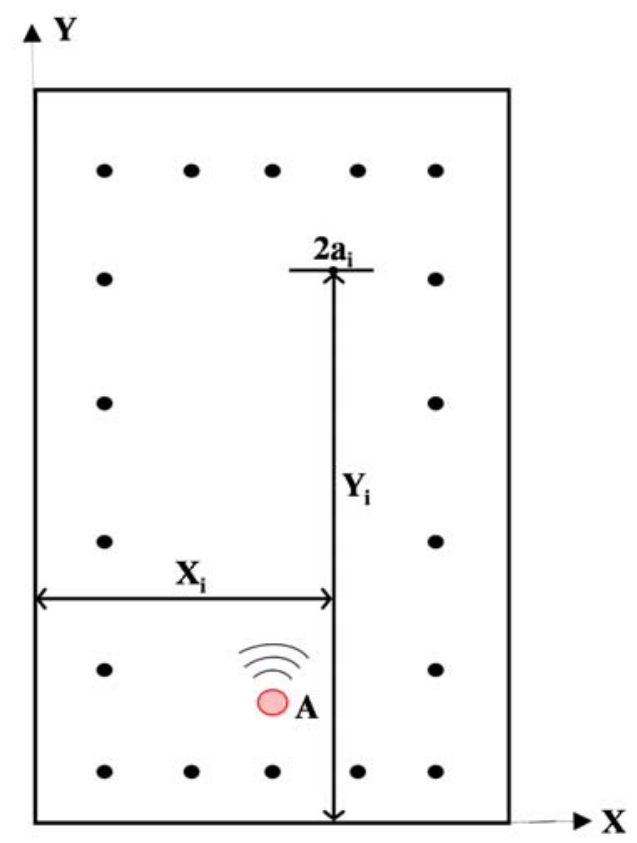

Figure 5. Assumed initial damage configuration. 

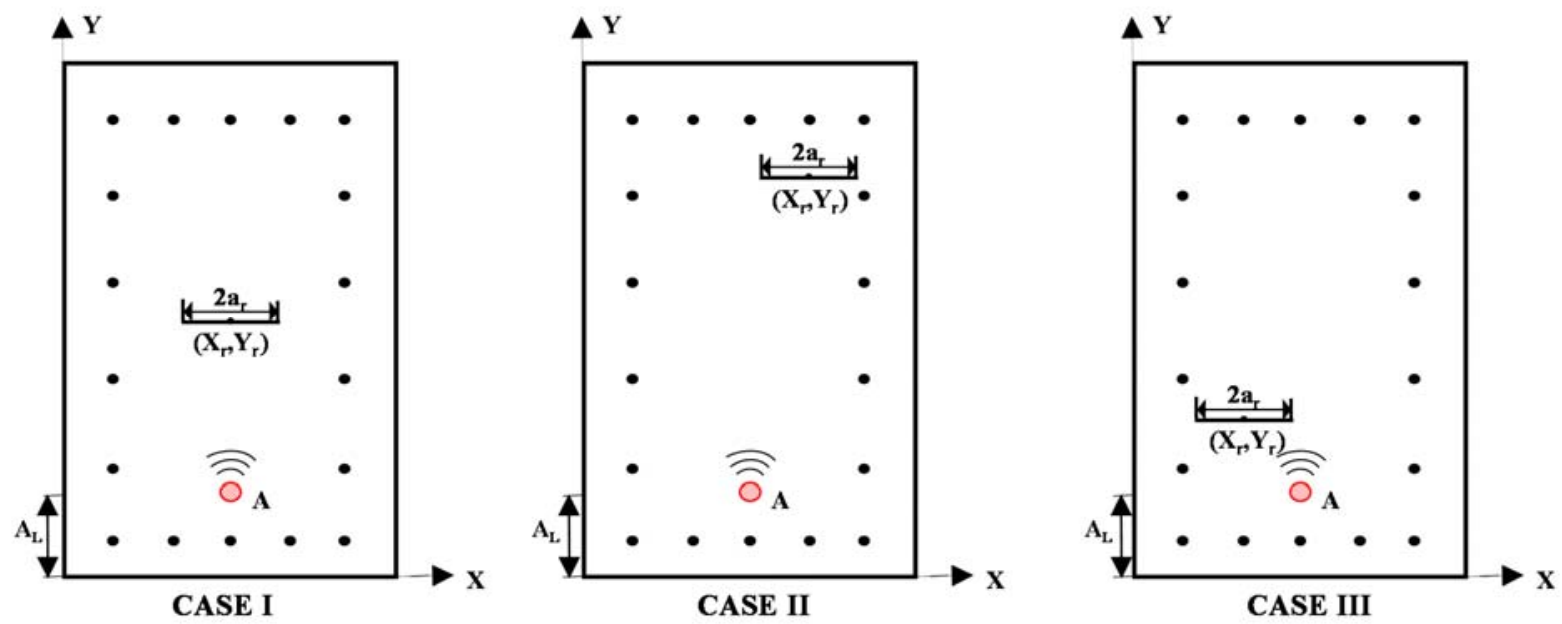

Figure 6. Three reference damage configurations considered in the example.

Case I-a center damage configuration: The GA based optimization procedure described earlier is initiated with an arbitrarily selected initial damage location within the upper and lower bounds specified. The damage location $\left(\mathrm{X}_{\mathrm{r}}, \mathrm{Y}_{\mathrm{r}}\right)$ variables have to be confined within upper and lower bounds. The final location of the damage is determined from the optimization procedure. The results from the optimization procedure are presented in Table 3. It can be seen from Table 3 that both $X_{r}$ and $Y_{r}$ locations of the damage are detected to within 1.5 percent.

Case II-a upper right damage configuration: The GA based optimization procedure described earlier is initiated with an arbitrarily selected initial damage location. The final location of the damage is determined from the optimization procedure. The results from the optimization procedure are presented in Table 3. It can be seen from Table 3 that both $X_{r}$ and $Y_{r}$ locations of the damage are detected to within 0.5 percent.

Case III-a bottom left damage configuration: The GA based optimization procedure described earlier is initiated with an arbitrarily selected initial damage location. The final location of the damage is determined from the optimization procedure. The results from the optimization procedure are presented in Table 3 . It can be seen from Table 3 that both $X_{r}$ and $Y_{r}$ locations of the damage are detected to within 1.0 percent.

Table 3: Different damage configurations: Two-unknown variables

\begin{tabular}{|c|c|c|c|c|c|c|}
\hline \multirow[b]{2}{*}{ Case } & \multicolumn{2}{|c|}{ Reference Configuration } & \multicolumn{2}{|c|}{ Optimization Result } & \multicolumn{2}{|c|}{$\begin{array}{l}\text { \% Difference from } \\
\text { Reference Solution }\end{array}$} \\
\hline & & & & & $\mathrm{X}_{\text {error }}$ & $Y_{\text {error }}$ \\
\hline $\mathrm{I}-\mathrm{a}$ & $0.500 \mathrm{w}$ & $0.500 \mathrm{~h}$ & $0.506 \mathrm{w}$ & $0.499 \mathrm{~h}$ & 1.20 & 0.20 \\
\hline II-a & $0.667 \mathrm{w}$ & $0.750 \mathrm{~h}$ & $0.665 w$ & $0.751 \mathrm{~h}$ & 0.29 & 0.13 \\
\hline III-a & $0.334 w$ & $0.250 \mathrm{~h}$ & $0.334 w$ & $0.252 \mathrm{~h}$ & 0.00 & 0.80 \\
\hline
\end{tabular}

\section{Damage Location and Size as the Unknown Variables}

For this problem, the locations, $X_{r}$ and $Y_{r}$, and the damage size, $2 a_{r}$, are treated as three unknowns in the optimization procedure as shown in Figure 6. The results from the analyses are described in the following section.

Case I-b center damage configuration: The GA based optimization procedure described earlier is initiated with an arbitrarily selected initial damage location and size within the upper and lower bounds specified. The final location of the damage and size is determined using the optimization procedure. The results from the optimization procedure are presented in Table 4 . It can be seen from Table 4 that damage location $\left(X_{r}, Y_{r}\right)$ and size $\left(2 a_{r}\right)$ are detected to within 4.5 percent.

Case II-b upper right damage configuration: The GA based optimization procedure described earlier is initiated with an arbitrarily selected initial damage location and size. The final location of the damage and size is 
determined using the optimization procedure. The results from the optimization procedure are presented in Table 4. It can be seen from Table 4 that damage location $\left(X_{r}, Y_{r}\right)$ and size $\left(2 a_{r}\right)$ are detected to within 3.0 percent.

Case III-b bottom left damage configuration: The GA based optimization procedure described earlier is initiated with an arbitrarily selected initial damage location and size. The final location of the damage and size is determined using the optimization procedure. The results from the optimization procedure are presented in Table 4. It can be seen from Table 4 that damage location $\left(X_{r}, Y_{r}\right)$ and size $\left(2 a_{r}\right)$ are detected to within 4.5 percent.

Table 4: Different damage configurations: Three-unknown variables

\begin{tabular}{|c|ccc|ccc|ccc|}
\hline & \multicolumn{2}{|c|}{ Reference Configuration } & \multicolumn{2}{c|}{ Optimization Result } & \multicolumn{3}{c|}{ \% Difference from } \\
Case & $\mathrm{X}_{\mathrm{r}}$ & $\mathrm{Y}_{\mathrm{r}}$ & $2 \mathrm{a}_{\mathrm{r}}$ & $\mathrm{X}_{\mathrm{f}}$ & $\mathrm{Y}_{\mathrm{f}}$ & $2 \mathrm{a}_{\mathrm{f}}$ & \multicolumn{2}{c}{ Reference Solution } \\
II-b & $0.500 \mathrm{w}$ & $0.500 \mathrm{~h}$ & $0.167 \mathrm{w}$ & $0.515 \mathrm{w}$ & $0.496 \mathrm{~h}$ & $0.160 \mathrm{w}$ & 3.00 & 0.80 & 4.19 \\
\hline II-b & $0.667 \mathrm{w}$ & $0.750 \mathrm{~h}$ & $0.167 \mathrm{w}$ & $0.659 \mathrm{w}$ & $0.757 \mathrm{~h}$ & $0.162 \mathrm{w}$ & 1.19 & 0.93 & 2.99 \\
\hline III-b & $0.334 \mathrm{w}$ & $0.250 \mathrm{~h}$ & $0.167 \mathrm{w}$ & $0.346 \mathrm{w}$ & $0.247 \mathrm{~h}$ & $0.160 \mathrm{w}$ & 3.59 & 1.20 & 4.21 \\
\hline
\end{tabular}

\section{Concluding Remarks}

In this paper, a damage characterization method using a wave propagation response estimated at different sensor locations is proposed. An optimization procedure combined with wave propagation analysis has been developed to predict damage size and location. It is demonstrated that the response estimated at various sensor locations can be effectively used to predict the damage location and size. Numerical examples were presented to demonstrate the GA optimization procedure in several damage configurations. First, the damage location was estimated keeping the damage size constant. It was found that the damage location was estimated to within one and one-half percent for the damage configuration tested. Next, both the damage size and the location were estimated. It was found that the size and location were estimated to within five percent for the configuration analyzed.

\section{References}

1. Kim, J. T., and Stubbs, N., “Improved Damage Identification Method Based on Modal Information,” Journal of Sound and Vibration, Vol. 252, pp. 223-238, 2002.

2. Mal, A. K., Ricci, F., Banerjee, S., and Shih, F., “A Conceptual Structural Health Monitoring System Based on Vibration and Wave Propagation,” Structural Health Monitoring: An International Journal, Vol. 4, pp. 283 - 293 , 2005.

3. Wang, L., and Yuan, F. G., “Active Damage Localization Technique Based on Energy Propagation of Lamb Waves,” Smart Structures and Systems, Vol. 3, pp. 201-217, 2007.

4. Yang, X. X., Chen, S. L., Jin, S. J., and Chang, W. S., "Crack Orientation and Depth Estimation in a LowPressure Turbine Disc using a Phased Array Ultrasonic Transducer and an Artificial Neural Network,”. Sensors, 13, pp. 12375-12391, 2013.

5. Jeanne, L. S., Kuo, T. W., Cheng, K. J., Chun, H. C., Jing, C. T., and Jiunn, W. L., “Applications of Flexible Ultrasonic Transducer Array for Defect Detection at 150 C,” Sensors, 13, pp. 975-983, 2013.

6. Castaings, M., Le, C. E., and Hosten, B., "Modal Decomposition Method for Modeling the Interaction of Lamb Waves with Cracks,” J. Acoust. Soc. Am., 112, pp. 2567-2582, 2002.

7. Olson, S. E., Derriso, M. M., DeSimio, M. P., and Thomas, D. T., “Analytical Modeling of Lamb Waves for Structural Health Monitoring,” Proceedings of the 3rd European Workshop on Structural Health Monitoring, Granada, Spain. 5-7 July, 2006. 
8. Krishnamurthy, T. and Gallegos, A. M., "Damage Characterization Using the Extended Finite Element Method for Structural Health Management,” AIAA, 2011.

9. Krishnamurthy, T., Hochhalter, J. D., and Gallegos, A. M., "Damage Characterization Method for Structural Health Management Using Reduced Number of Sensor Inputs,” AIAA, 2012.

10. Mal, A., “Structural Health Monitoring,” American Academy of Mechanics, Vol 33, No 11-12, pp. 1-4, 2004.

11. Krautkramer, J., “Emerging Technology - Guided Wave Ultrasonics,” NDTnet, Vol 3, No 6, 1998.

12. Wait, J., R., Park, G., and Farrar, C., R., "Integrated Structural Health Assessment Using Piezoelectric Active Sensors,” Shock and Vibration, 12, pp. 389-405, 2005.

13. Castaings, M., Le, C. E., and Hosten, B., "Modal Decomposition Method for Modeling the Interaction of Lamb Waves with Cracks,” J. Acoust. Soc. Am., 112, pp. 2567-2582, 2002.

14. Le, C. E., Castaings, M., and Hosten, B., "The Interaction of the $\mathrm{S}_{0}$ Lamb Mode with Vertical Cracks in an Aluminum Plate,” Ultrasonics, 40, pp. 187-192, 2002.

15. Wang, L. G., and Shen, J. Z., “Scattering of Elastic Waves by a Crack in an Isotropic Plate,” Ultrasonics, 35, pp. 451-457, 1997.

16. Lu, Y., Ye, L., Su, Z. Q., and Yang, C. H., “Quantitative Assessment of Through-Thickness Crack Size Based on Lamb Wave Scattering in Aluminum Plates,” NDT E Int. 41, pp. 59-68, 2008.

17. Dutta, D., Sohn, H., and Harries, K. A., “A Nonlinear Acoustic Technique for Crack Detection in Metallic Structures,” Struct. Health Monit., 8, pp. 251-262, 2009.

18. Soshu H., and Toshihiko S., "Detection of a Closed Crack by Nonlinear Acoustics using Ultrasonic Transducers,” Review of Progress in Quantitative Nondestructive Evaluation, 32nd ed.; Thompson, D. O., Ed.; Book News, Inc.: New York, NY, pp. 277-282, 2006.

19. Shen, Y., and Giurgiutiu, V., “An Analytical Model for PWAS-generated 2D Ultrasonic Guided Wave Propagation ,” Proceedings of the Health Monitoring of Structural and Biological Systems Conference, San Diego, CA, March 2012.

20. Shen Y., and Giurgiutiu V., "Predictive Modeling of Nonlinear Wave Propagation for Structural Health Monitoring with Piezoelectric Wafer Active Sensors,” J. Intell. Mater. Syst. Struct., 25, pp. 506-520, 2014.

21. Wan, X., Zhang, Q., Xu. G. and Tse. P. W., "Numerical Simulation of Nonlinear Lamb Waves used in a Thin Plate for Detecting Buried Micro-Cracks,” Sensors, 14, pp. 8528-8546, 2014.

22. Jia, X., "Model Analysis of Lamb Wave Generation in Elastic Plates by Liquid Wedge Transducers," Acoustical Society of America, Vol 101, No 2, pp. 834-842, February, 1997.

23. ABAQUS Documentations, Version 6.12, ABAQUS, Inc., Providence, RI.

24. Shen, Y., and Giurgiutiu, V., "Predictive Simulation of Nonlinear Ultrasonics.” Proceedings of the Health Monitoring of Structural and Biological Systems Conference, San Diego, CA, March 2012.

25. Moser, F., Jacobs, L.J., and Qu, J., "Modeling Elastic Wave Propagation in Waveguides with the Finite Element Method,” NDT\&E International, 32(4), pp. 225-234, 1999.

26. Math Works, http://www.mathworks.com/help/toolbox/ga/.

27. Python, http://www.python.org/home/. 